\title{
Ubiquitin-specific protease 3 promotes cell migration and invasion by interacting with and deubiquitinating SUZ12 in gastric cancer
}

Xiaosheng $\mathrm{Wu}^{1}$, Mengwei Liu ${ }^{1}$, Huiqiong Zhu ${ }^{1}$, Jing Wang ${ }^{1}$, Weiyu Dai ${ }^{1}$, Jiaying Li ${ }^{1}$, Danping Zhu ${ }^{2}$, Weimei Tang ${ }^{1}$, Yizhi Xiao ${ }^{1}$, Jianjiao Lin ${ }^{3}$, Wenjing Zhang ${ }^{4}$, Yong Sun ${ }^{1}$, Yi Zhang ${ }^{1}$, Yaying Chen ${ }^{5}$, Guoxin Li ${ }^{6}$, Aimin Li ${ }^{1}$, Li Xiang ${ }^{3,7^{*}}$, Side Liu ${ }^{1,3,7^{*}}$ and Jide Wang ${ }^{1,3,7^{*}}$

\begin{abstract}
Background: The deubiquitinating enzyme ubiquitin-specific protease 3 (USP3) plays a crucial role in numerous biological processes. The aberrant expression of USP3 may have an important role in tumor development. However, the mechanism by which USP3 promotes gastric cancer (GC) metastasis remains largely unknown.

Methods: Effects of USP3 on the progression of GC in vivo and in vitro and the potential underlying mechanisms have been investigated utilizing proteomics, RT-PCR, western blotting, immunohistochemistry, immunofluorescence, cell invasion and migration assays and xenograft tumor models.

Results: USP3 expression was upregulated in GC compared with matched normal tissues and was predictive of poor survival. USP3 also promoted migration and epithelial-to-mesenchymal transition (EMT) in GC cells. Moreover, TGF- $\beta 1$ induced USP3 expression, and USP3 knockdown inhibited TGF- $\beta 1$-induced EMT. Furthermore, we utilized Isobaric Tag for Relative and Absolute Quantitation (iTRAQ) to identify differentially expressed proteins in USP3-overexpressing cells compared with control cells. Importantly, we found that SUZ12 is indispensable for USP3-mediated oncogenic activity in GC. We observed that USP3 interacted with and stabilized SUZ12 via deubiquitination. SUZ12 knockdown inhibited USP3-induced migration and invasion, as well as EMT in GC cells. Examination of clinical samples confirmed that USP3 expression was positively correlated with SUZ12 protein expression and that the levels of USP3 or SUZ12 protein were negatively correlated with the levels of E-cadherin protein.
\end{abstract}

Conclusions: These findings identify USP3 as a critical regulator. The USP3-SUZ12 axis might promote tumor progression and could be a potential therapeutic candidate for human GC.

Keywords: USP3, SUZ12, Gastric cancer, EMT, iTRAQ

\footnotetext{
*Correspondence: shellyxiang@sina.com; liuside@163.com;

jidewang55@163.com

${ }^{3}$ Department of Gastroenterology, Longgang District People's Hospital,

Shenzhen 518172, China

${ }^{1}$ Guangdong Provincial Key Laboratory of Gastroenterology, Department of

Gastroenterology, Nanfang Hospital, Southern Medical University, Guangzhou

510515, China

Full list of author information is available at the end of the article
}

(C) The Author(s). 2019 Open Access This article is distributed under the terms of the Creative Commons Attribution 4.0 International License (http://creativecommons.org/licenses/by/4.0/), which permits unrestricted use, distribution, and reproduction in any medium, provided you give appropriate credit to the original author(s) and the source, provide a link to the Creative Commons license, and indicate if changes were made. The Creative Commons Public Domain Dedication waiver (http://creativecommons.org/publicdomain/zero/1.0/) applies to the data made available in this article, unless otherwise stated. 


\section{Background}

Gastric cancer (GC) is the fifth most common malignancy and the third leading cause of cancer-related deaths worldwide [1]. GC metastasis, which is responsible for almost $90 \%$ of the lethality among GC patients, is a multistep process that results from alterations in genes, including the activation of oncogenes and the inactivation and mutation of tumor suppressor genes [2]. Through greater awareness, earlier detection, and advances in treatment, the survival of GC patients has improved; however, a proportion of patients will eventually succumb to the disease as a result of metastasis [3]. Therefore, the identification of novel genes and the discovery of the detailed mechanisms underlying GC metastasis are urgently needed.

The ubiquitin-proteasome pathway is an important intracellular protein degradation regulatory system [4]. Deubiquitination is an important opposing mechanism of ubiquitination and is catalyzed by deubiquitinating enzymes (DUBs). Approximately 100 DUBs have been reported to be encoded by human genes and are divided into 6 subclasses based on their Ub-protease domains [5, 6]. The ubiquitin-specific proteases (USPs) are the largest subclass of DUBs [7]. The USP3 gene is a member of the USP family. Recent progress has highlighted the significance of USP3 in tumor progression. For example, in one study, USP3 was overexpressed in GC tissues and cells, and ectopic USP3 promoted GC tumor growth [8]. USP3 participates in the reverse process of ubiquitination by removing ubiquitin from target proteins and rescuing proteins that are marked for degradation [9]. USP3 was reported to deubiquitinate RIG-1-like receptors and to inhibit type I interferon signaling [10]. However, it is unclear whether USP3 modulates target proteins to regulate cell migration and invasion in GC.

SUZ12 (Suppressor of zeste 12 protein homolog) is a core component of the Polycomb PRC2-HMTase complex, which has been shown to be involved in stem cell maintenance and development [11, 12]. SUZ12 is upregulated in many human cancers including colon [13], liver [14], gastric [15] and breast cancers [16]. For example, SUZ12 expression was significantly increased in gastric tumor tissues compared with normal tissues [15]. SUZ12 expression was found to be associated with pathological stage, metastasis distance, and shorter overall survival of GC patients. Moreover, SUZ12 induced tumor cell epithelial-to-mesenchymal transition (EMT) and maintained the invasive potential of cancer, which appeared to play a crucial role in the metastatic progression of human carcinomas [16, 17].

Based on these findings, we further investigated the molecular mechanism by which USP3 regulates GC progression. In this study, we found that TGF- $\beta$-induced USP3 promotes EMT and metastasis. We also demonstrated that USP3 is a positive regulator of SUZ12 and that it deubiquitinates and stabilizes SUZ12 and promotes progression of GC.

\section{Materials and methods \\ Cells culture, antibodies and reagents}

See Additional file 1: Supplementary materials and methods.

\section{Tissue multi-array (TMA) and immunohistochemistry}

The GC samples and the corresponding adjacent nontumor tissues were used to construct a tissue microarray (TMA; Shanghai Biochip Co., Ltd., Shanghai, China). The tissue microarray was stained for USP3 expression. The TMA was scored independently by two pathologists for both staining intensity and extent of protein expression across the section. Immunohistochemical staining was performed using the Dako Envision Plus System (Dako, Carpinteria, CA, USA) according to the manufacturer's instructions. Analysis was performed by two independent observers who were blinded to the clinical outcome. Staining intensity was scored as 0 , negative staining; 1 , weak staining; 2, moderate staining; and 3, intense staining. Cells with negative or weak staining were defined as low expressers, and cells with moderate or intense staining were defined as high expressers, as previously described [18]. This study was approved by the institutional human ethics committee of the relevant institutions.

\section{Plasmid construction and small interfering RNA transfection}

The USP3 or SUZ12 coding sequence (CDS) was cloned into the pENTER shuttle plasmid (ViGene Biosciences Inc., Rockville, MD, USA). pENTER- USP3 and SUZ12 and empty vector (pENTER) plasmids encoding FLAG or MYC tag were purchased from Vigene. Populations of pENTER vector and pENTER USP3 or SUZ12 pooled stable transfectants of GC lines were obtained using the same plasmid and selection process as described previously [19].

For RNA interference studies, GC cells were transfected with predesigned small interfering RNAs (siRNAs) at final concentrations of $25 \mathrm{nM}$. The siRNA sequences were as follows: USP3 sense strand: siRNA 1: TTCACAGCTGACAGGCATA, siRNA 2: CCTTCAGTCACTCAGTAAC, siRNA 3: CCATGAATTCATGCGCTAC; SUZ12 sense strand: siRNA 1: GTCTCATCGAAACTCCAGA. Src siRNA, 5'-TTCTCCGAACGTGTCACGT-3', which did not target any genes, was used as the negative control. The cells were transfected with siRNA duplexes using oligofectamine (Invitrogen-Life Technologies) according to the manufacturer's instructions. The effect of gene knockdown was evaluated by western blotting $48 \mathrm{~h}$ post transfection. 
Co-immunoprecipitations and Western blotting analysis

See Additional file 1: Supplementary materials and methods.

\section{Quantitative RT-qPCR}

See Additional file 1: Supplementary materials and methods.

\section{Wound-healing assays}

See Additional file 1: Supplementary materials and methods.

\section{Invasion assays}

See Additional file 1: Supplementary materials and methods.

Immunofluorescence staining and confocal laser scanning microscopy

See Additional file 1: Supplementary materials and methods.

\section{Protein extraction and preparation for isobaric tag for relative and absolute quantitation (iTRAQ)}

The BGC-823 cells were lysed in lysis buffer $(20 \mathrm{mM}$ Tris- $\mathrm{HCl}, \mathrm{pH} 7.5,150 \mathrm{mM} \mathrm{NaCl}, 1 \mathrm{mM}$ EDTA, and 1\% Triton X-100 with protease inhibitor) and sonicated, and the supernatant was collected by centrifugation at 30, $000 \mathrm{~g}$ for $15 \mathrm{~min}$. After treatment with acetone, the cell extracts were resuspended in $250 \mathrm{mM}$ triethylammonium bicarbonate (TEAB), which was followed by incubation with $10 \mathrm{mM}$ DL-dithiothreitol (DTT) in a water bath at $56^{\circ} \mathrm{C}$ for $1 \mathrm{~h}$, and then with $55 \mathrm{mM}$ iodoacetamide (IAM) in the dark at room temperature for $45 \mathrm{~min}$. The proteins $(100 \mu \mathrm{g}$ each) were digested in trypsin at $37^{\circ} \mathrm{C}$ for $16 \mathrm{~h}$. Subsequently, the peptides were dried by vacuum centrifuge and resuspended in $500 \mathrm{mM}$ TEAB for further iTRAQ labeling. The protein concentration was determined using a Bradford assay.

\section{ITRAQ labeling and strong cation exchange (SCX) separation}

The iTRAQ labeling of tryptic peptides was performed using an iTRAQ reagent Multiplex kit (Applied Biosystems, Foster City, CA, USA) according to the manufacturer's protocol. The peptides labeled with respective isobaric tags were incubated at room temperature for $2 \mathrm{~h}$ and vacuum centrifuged until dry. Then, the iTRAQlabeled peptide samples were reconstituted in Buffer A [25 $\mathrm{mM} \mathrm{NaH}_{2} \mathrm{PO}_{4}, 25 \%$ acetonitrile $(\mathrm{ACN}), \mathrm{pH}$ 2.7] and fractionated using an Ultremex SCX column $(250 \times 4.6$ $\mathrm{mm}, 5 \mu \mathrm{m}$ particle size, $200 \AA$ pore size) via an LC-20AB HPLC pump system (Shimadzu, Japan) at a flow rate of $1.0 \mathrm{ml} / \mathrm{min}$. The $35 \mathrm{~min}$ HPLC gradient consisted of $100 \%$ Buffer A for $10 \mathrm{~min}, 5-35 \%$ Buffer B (25 mM NaH${ }_{2} \mathrm{PO}_{4}$,
25\% ACN, $1 \mathrm{M} \mathrm{KCl}, \mathrm{pH} 2.7)$ for $11 \mathrm{~min}, 35-80 \%$ Buffer B for $1 \mathrm{~min}$, 80\% Buffer B for 3 min and 100\% Buffer A for $10 \mathrm{~min}$. The chromatograms were recorded at $214 \mathrm{~nm}$. The collected fractions were desalted using a Strata X C18 column (Phenomenex), vacuum centrifuged and reconstituted in $0.1 \%$ formic acid (FA) for subsequent LC-MS/ MS analysis.

\section{Mass spectrometric analysis}

Samples were analyzed on a Thermo Scientific Q Exactive mass spectrometer (Fitgene Biotechnology CO., LTD, Guangzhou, China). The peptides were subjected to nanoelectrospray ionization (NSI) followed by tandem mass spectrometry (MS/MS) in a QEXACTIVE (Thermo Fisher Scientific, San Jose, CA) coupled online to the HPLC. Protein identification was performed with MASCOT software by searching UniProt_Aedis. Differentially expressed proteins were filtered for a fold change cutoff of 1.2 and a $p$-value cutoff of 0.05 . The experiments were repeated three times.

\section{Cycloheximide treatment}

To determine the stability of endogenous SUZ12 protein, cells were transfected with either empty plasmid or the pENTER-FLAG-USP3 plasmid with $100 \mu \mathrm{M}$ cycloheximide for the times indicated in the figure legends. Total protein amounts of cell lysates were determined by BCA assay. Lysates with equivalent protein amounts were resolved by SDS-PAGE and analyzed by Western blotting to determine the abundance of endogenous SUZ12 protein.

\section{In vitro deubiquitylation assay}

Deubiquitination assays were conducted as previously described $[9,10]$. In brief, for analyzing the ubiquitination of SUZ12, BCG-823 cells was transfected with Flag-USP3 or vector for $48 \mathrm{~h}$. Cell lysates were prepared with cell lysis buffer. Whole-cell lysates were immunoprecipitated with the indicated antibodies (anti-SUZ12 or IgG) on protein A/ $\mathrm{G}$ beads (Santa Cruz Biotechnology) overnight. The beads were then washed and boiled in SDS loading buffer. Immunoprecipitated protein complexes ((anti-SUZ12, anti-USP3, anti-poly-UBC and anti-GAPDH) were assessed using Western blotting.

\section{Protein degradation assay}

MG132 (carbobenzoxy-Leu-Leu-leucinal) was purchased from Sigma-Aldrich. MG132 is a peptide aldehyde, which effectively blocks the proteolytic activity of the $26 \mathrm{~S}$ proteasome complex. We measured the SUZ12 expression level following treatment with MG132 under different USP3 conditions to confirm whether USP3 inhibits SUZ12 degradation. 


\section{Construction of lentivirus and orthotopic mouse metastatic model}

Lentivirus expressing EGFP/USP3 (LV- USP3) was constructed by Genechem (Shanghai, China) using UbiMCS-3FLAG-CBh-gcGFP-IRES-puromycin vector. UbiMCS-3FLAG-CBh-gcGFP-IRES-puromycin empty vector were used as controls. Selected pools of over expressing cells were used for subsequent experiments.

A surgical orthotopic implantation mouse model of GC was performed as described previously [20]. A single-cell suspension of $5 \times 10^{6}$ BCG-823/pEGFP/vector and BCG823/EGFP/USP3 -transduced cells/100 $\mu$ l PBS was injected via the tail vein. The progression of cancer cell growth was monitored after 34 days by bioluminescent imaging using the IVIS100 Imaging System (Kodak, Rochester, NY, USA). Before surgical and analytical procedures were performed, the mice were anesthetized. The metastatic tissues were analyzed by hematoxylin and eosin staining, immunohistochemistry (IHC) and qRT-PCR. The Committee on the Use of Live Animals in Teaching and Research, the Southern Medical University,China, approved the protocol (authorization protocol number: NFEC-2017-062).

\section{Statistical analysis}

Statistical analyses were performed using the SPSS statistical software package (standard version 20.0 PSS, Chicago, IL). Quantitative data obtained from experiments with biological replicates are shown as the mean \pm standard deviation. Linear regression and Pearson correlation analysis were performed. Survival analysis was performed with the Kaplan-Meier and log-rank tests. Two-tailed Student's ttest was used to analyze the quantitative data with significant difference being considered if $P$ values was $<0.05$.

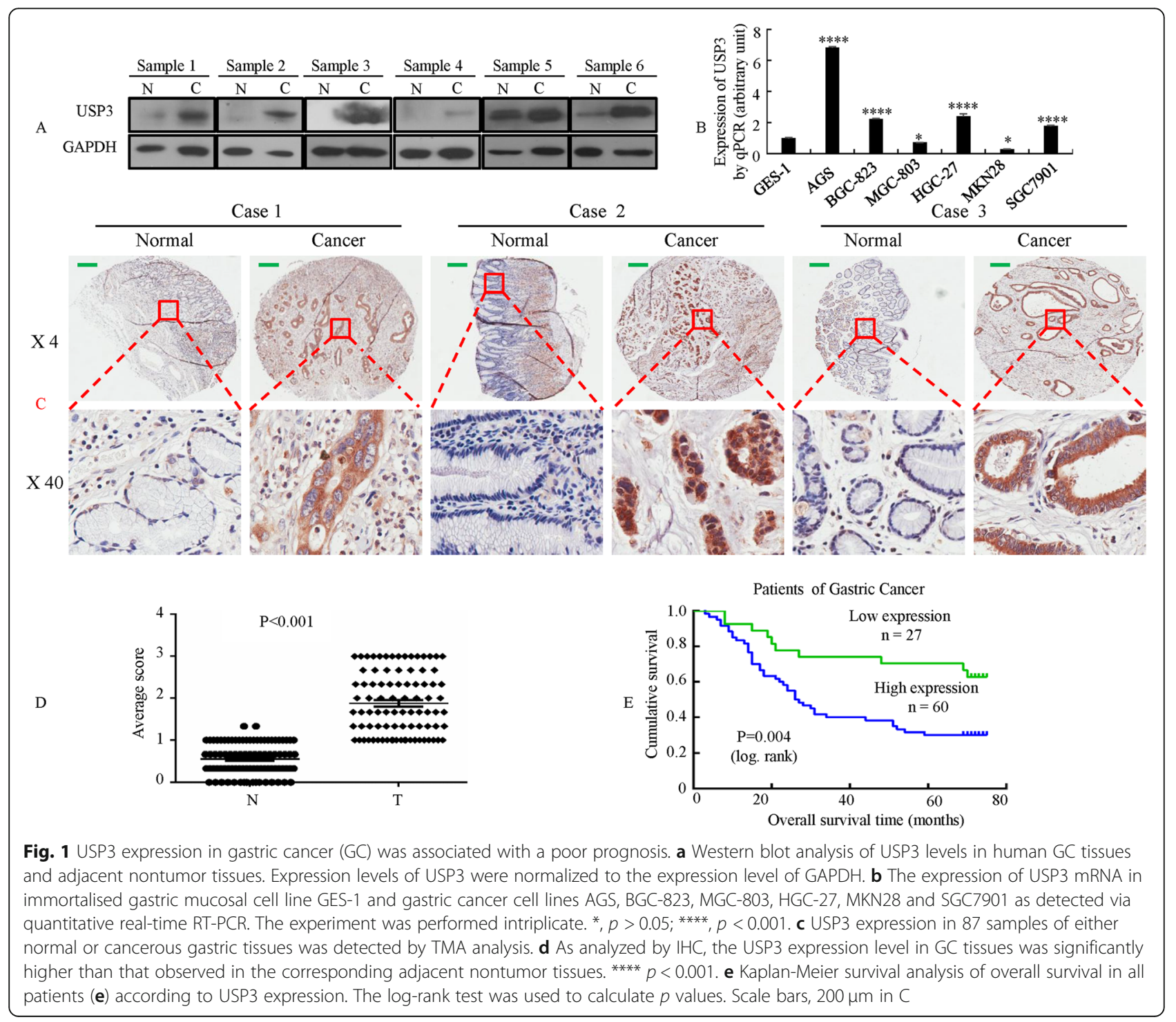




\section{Results}

Increased USP3 expression is correlated with tumor progression and poor prognosis in GC patients

To determine the role of USP3 in the aggressiveness of GC, we investigated the expression levels of USP3 by Western blot in 6 pairs of GC tissues. The results showed that USP3 protein was upregulated in GC tissues compared with adjacent nontumor tissues (Fig. 1a). Next, the expression of USP3 was detected in six cancer cell lines by semiquantitative RT-PCR assay. As shown in Fig. 1b, the GC cell lines AGS, BGC-823, HGC-27 and SGC-7901 showed elevated expression of USP3, while MGC-803 and MKN28 did not demonstrate increased USP3 expression levels compared with human gastric epithelial cell line GES-1.

Moreover, USP3 expression was analyzed in 87 GC tissue samples and was compared with the expression in adjacent nontumor tissues by tissue microarray (TMA). The human GC tissues exhibited greater immunostaining, whereas the normal gastric tissues exhibited less immunostaining (Fig. 1c). Semiquantitative scoring showed that USP3 protein was expressed at significantly higher levels in cancer tissues compared with adjacent nontumor tissues (Fig. 1d).

Clinicopathologic analysis revealed that expression of USP3 was positively correlated with tumor differentiation status $(P<0.001)$, lymph node metastasis $(P=$ $0.013)$, tumor size $(<10 \mathrm{~cm}$ vs $\geq 10 \mathrm{~cm}, P=0.016)$, AJCC T stage (I/II vs. III/IV, $P=0.029)$, and clinical TNM stage (I/II vs. III/IV, $\mathrm{P}<0.001)$. USP3 staining did not significantly correlate with age $(P=0.383)$ or gender $(P=$ 0.808) (Additional file 1: Table S1).

The overall survival rate of GC patients with high USP3 expression was significantly poorer than that of patients with low USP3 expression by the Kaplan-Meier method $(P=0.004$; Fig. $1 \mathrm{e})$.

Collectively, these results suggested that USP3 may play a role in GC development and progression.

\section{Upregulation of USP3 promotes metastasis through EMT in GC}

Elevated cell migration and invasion are associated with the increased metastatic potential of cancer cells [21, 22 ], which may be independent of cell proliferation rates. Therefore, we studied the effect of USP3 on cell invasion and migration of MGC-803 (Low-level expression, Fig. 1b) and AGS and BGC-823 (High-level expression, Fig. 1b) cell lines using the transwell and wound-healing assay. The data showed that ectopic expression of USP3 promoted GC cells invasion and migration compared with the vector control cells (Fig. 2a-c). Moreover, the AGS and BGC-823 cells showed higher invasion and migration rates compared to the MGC-803 cells (Fig. 2a-c, Additional file 2: Figure S1A-C). Then, we synthesized 3 pairs of USP3 siRNA (pool siRNA oligonucleotides). We showed that knock-down of USP3 could inhibit the invasive and migration abilities of AGS and BGC-823 cells (Fig. 2d \& e; Additional file 2: Figure S1D \& E). These results suggest that high-level expression of USP3 may contribute to the metastasis of GC by promoting the invasion and migration ability of GC cells.

The acquisition of an EMT phenotype is a critical process for switching early stage carcinomas into invasive malignancies, which is often associated with the loss of epithelial differentiation and gain of mesenchymal phenotype [20, 23]. We next examined the morphologic features of GC cells. The stable vector-transfected AGS and BGC-823 cells exhibited a cobblestone-like typical epithelial morphology and were present as a confluent monolayer or as islands of grouped cells with tight cell-cell contacts. However, the USP3-transfected cells underwent a striking morphologic change, which was characterized by loss of cell-cell contacts and a cobblestone-like phenotype, failure to form a confluent monolayer or islands, and the gain of an elongated and spindle-shaped morphology and scattered appearance. These phenotypes are typical of the fibroblastoid cells generated during the EMT process (Fig. 2f).

Expression of epithelial and mesenchymal molecular markers was confirmed by immunofluorescence and western blot. Expression of epithelial markers, E-cadherin, was significantly reduced in USP3 transfectants cells. In contrast, expression of mesenchymal markers, vimentin (which positively correlate with EMT), was dramatically upregulated in USP3 transfectants cells, and vice versa (Fig. $2 \mathrm{~g} \& \mathrm{~h}$ ).

Several studies have shown that phosphorylation level proteins which involved ERKl/2/Akt signaling has emerged as a central feature of EMT, which is the initial step for metastasis in many cancer models [19, 22]. We performed western blot analysis to elucidate the phosphorylation status of proteins. We showed that the phosphorylation levels of AKT and ERK1/2 increased in USP3overexpressing cells; while the level of p-AKT and ERK1/2 decreased siRNA-mediated knockdown of USP3 in GC cells. The total amount of AKT and ERK1/2 protein was unaltered (Additional file 2: Figure S1F). Moreover, the expression level of the typical EMT epithelial marker Ecadherin was downregulated in USP3-overexpressing cells and vice versa (Additional file 2: Figure S1F).

Taken together, these observations suggest that the overexpression of USP3 facilitates gastric cancer invasion and metastasis through inducing EMT program.

\section{USP3 is required for TGF- $\beta 1$-induced EMT and cell migration in GC}

Several studies have reported that TGF- $\beta$ induces EMT in GC cells $[24,25]$. To investigate whether TGF- $\beta$ affects USP3 expression, we first examined the levels of USP3 in TGF- $\beta$-treated BCG-823 cells. Notably, TGF- $\beta$ treatment 


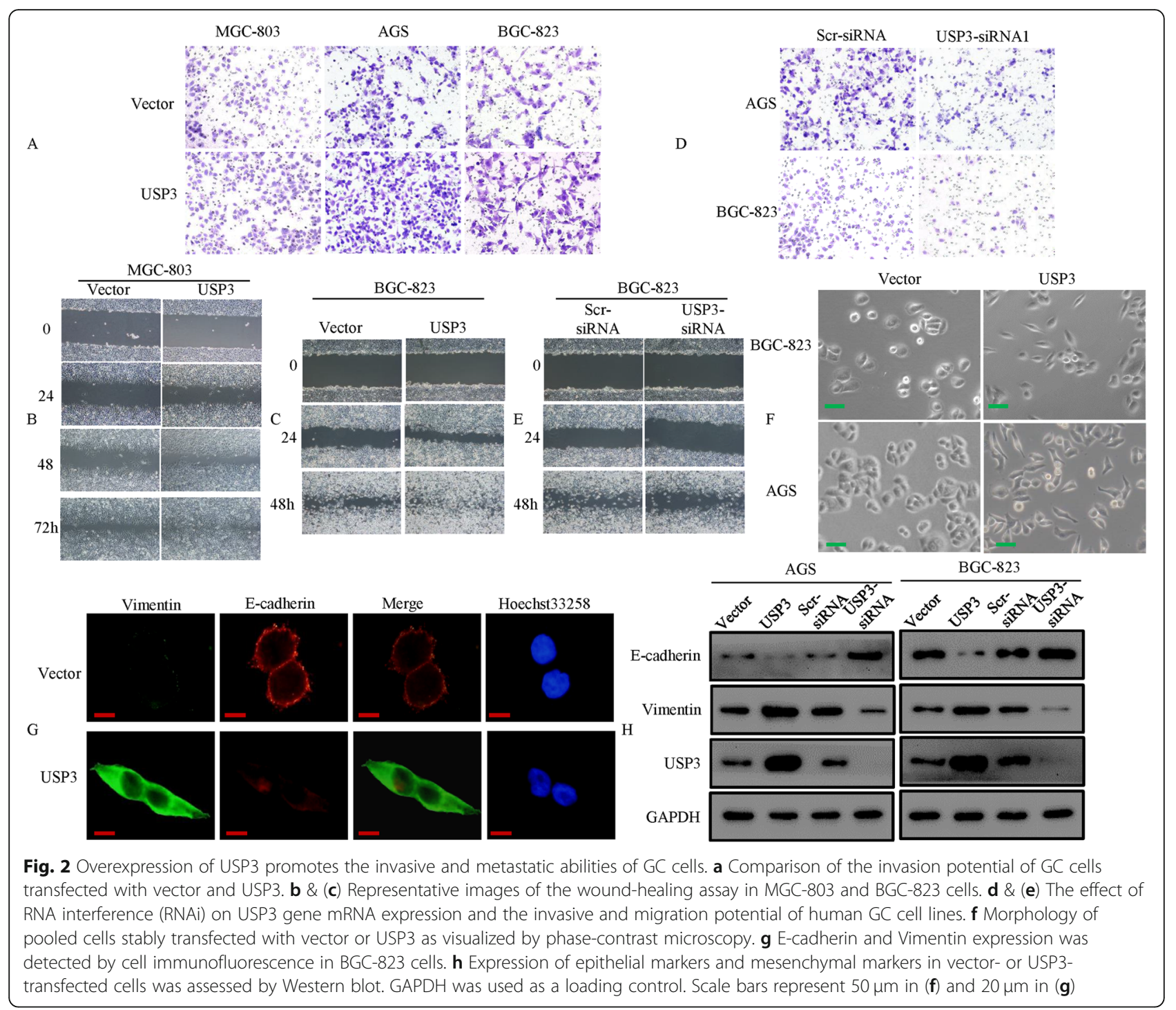

resulted in a significant increase in USP3 expression in a dose- and time-dependent manner. Moreover, TGF- $\beta 1$ markedly inhibited the expression of the EMT marker Ecadherin (Additional file 3: Figure S2A \& B). Blocking TGF- $\beta 1$ with a neutralizing antibody significantly suppressed endogenous USP3 expression and TGF- $\beta 1$ induced USP3 expression in BGC-823 cells (Additional file 3: Figure S2C). However, pretransfecting cells with USP3 siRNA not only inhibited USP3 expression but also suppressed TGF- $\beta 1$-induced vimentin expression (Additional file 3: Figure S2D). In addition, the results indicated that USP3 siRNA neutralized the influence of TGF- $\beta 1$ on cell phenotypes (Additional file 3: Figure S2E). Furthermore, downregulation of USP3 significantly antagonized the TGF- $\beta$-induced invasiveness of GC cells (Additional file 3: Figure S2F).

These experiments suggest that USP3 is involved in and required for TGF- $\beta$-induced EMT.

\section{USP3 promotes EMT and metastasis in vivo}

To test the effects of USP3 on GC metastasis in vivo, the Lv-vector or Lv-USP3 cells were injected into the tail vein of nude mice. On day 34 after inoculation, the mice were sacrificed, the lungs were collected, and metastatic colonies were counted. The USP3-overexpressing cells formed a variety of large metastatic nodules in the lungs compared with the vector cells (Fig. 3a). The mean number of metastatic colonies in the USP3-overexpressing cells group indicated enhanced invasion compared with that in the vector cells (Fig. 3b). The presence of lung metastases from BGC-823 cells was confirmed by histological analysis (Fig. 3c).

We next tested whether USP3 affects the EMT process. Using qPCR, we found that USP3-overexpressing cells resulted in a substantial decrease in E-cadherin expression compared with vector cells (Fig. 3d). Additionally, the presence of GC metastases in the lungs was confirmed by IHC using anti-E-cadherin antibodies (Fig. 3e). 
These findings suggest that ectopic USP3 expression is involved in the regulation of EMT and metastasis in GC cells.

\section{USP3 can influence the expression of SUZ12 protein in GC cells}

To determine differentially expressed proteins that may serve as potential interaction targets of USP3, total protein extracted from the treated BGC-823 cells stably expressing the Flag-USP3 fusion protein and the BGC-823 cells carrying an empty vector was analyzed using iTRAQ experiments. In total, 5631 proteins (29,570 unique peptides) were confidently identified and were used for further quantification analysis. Using the criteria described in the "Material and methods", 63 proteins were found to be downregulated (Additional file 1: Table S2) and 59 were found to be upregulated in the treated BGC-823 cells compared with the control cells (Table 1). Among them, SUZ12 was upregulated when the USP3 gene was ectopically expressed. Real-time PCR analysis suggested that overexpression of USP3 or the knock down of USP3 by siRNA had no effect on the SUZ12 mRNA level (Fig. 4a). However, according to Western blot analysis, endogenous SUZ12 was increased upon USP3 upregulation in GC cells (Fig. 4b) and reduced upon USP3 inhibition (Fig. 4c), which indicates that the regulatory effects of USP3 on SUZ12 expression only occur at the posttranscriptional level.

Furthermore, the localization of USP3 and SUZ12 was visualized by confocal laser scanning microscopy. The results demonstrated that both proteins were localized to the same regions in GC cells. Overlapping fluorescence signals revealed that the two proteins were colocalized mainly in the nucleus and slightly in the cytoplasm (Fig. 4d).

Together, these findings indicate that USP3 should be a potent regulator of SUZ12 in GC cells.

\section{USP3 interacts with and deubiquitinates SUZ12}

To elucidate the mechanisms responsible for the effects of USP3 on SUZ12, GC cells were cotransfected with Flagtagged USP3 and Myc-tagged SUZ12 constructs. We found that Flag-tagged USP3 could be co-immunoprecipitated with Myc-tagged SUZ12 in GC cells. Similarly, Myc-tagged SUZ12 was co-immunoprecipitated using an anti-USP3 (Flag) antibody (Fig. 5a). To further confirm that USP3 was associated with the SUZ12 protein, we performed reciprocal co-immunoprecipitation experiments using endogenous protein and showed that USP3 can interact with SUZ12 (Fig. 5b). Collectively, these findings suggest that USP3 can physically interact with SUZ12.

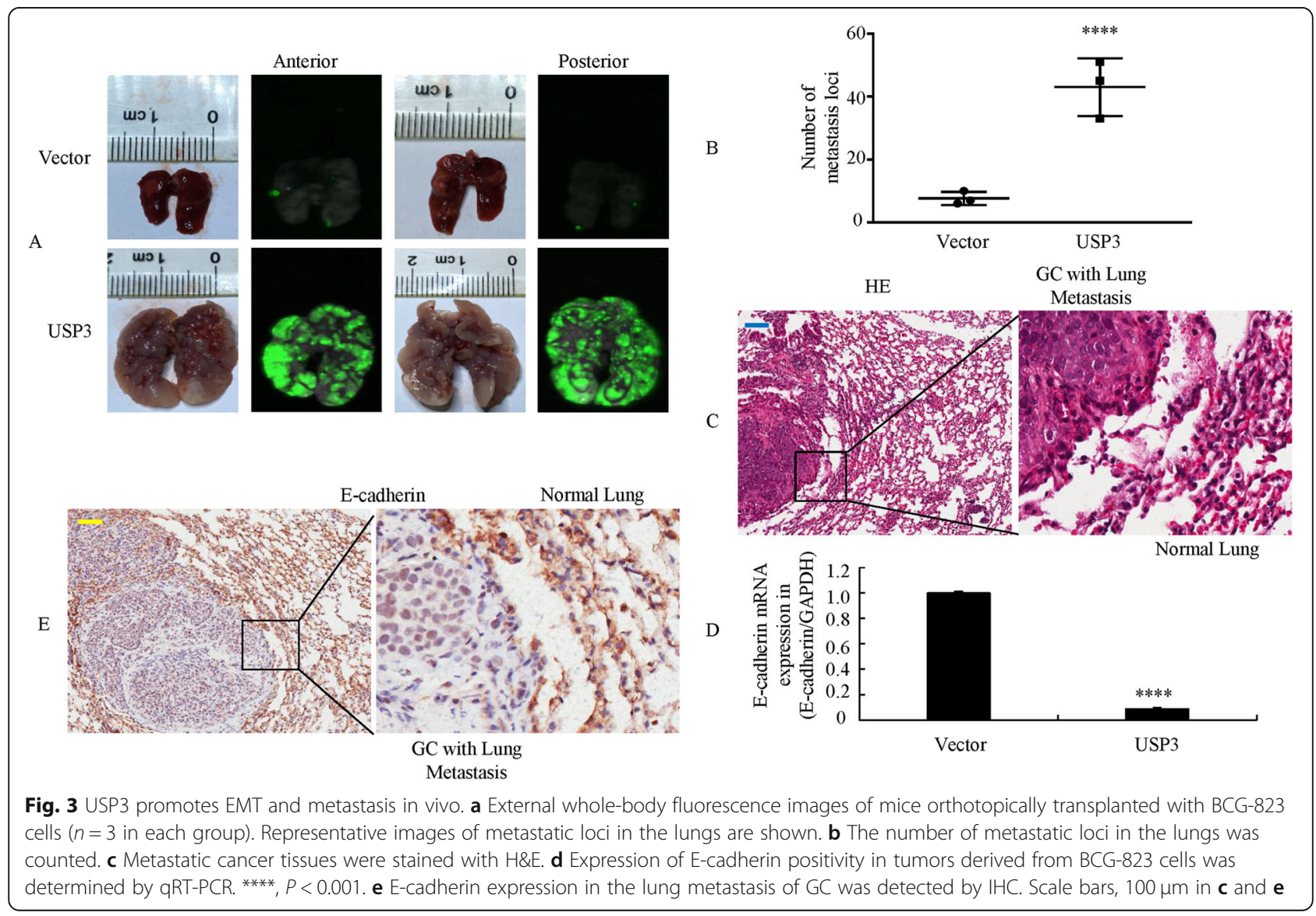


Table 1 iTRAQ ratios of up-regulated proteins in GC tissues

\begin{tabular}{|c|c|c|c|c|c|}
\hline No. & Protein name & Gene name & Accession Number & Molecular Weight & Ratio \\
\hline 1 & Muskelin & MKLN1 & gi|31,881,798 & $85 \mathrm{kDa}$ & 2.23 \\
\hline 2 & Polypeptide N-acetylgalactosaminyltransferase 13 & GLT13 & gi|145,309,313 & $48 \mathrm{kDa}$ & 2.06 \\
\hline 3 & Keratin, type II cytoskeletal 1 & $\mathrm{~K} 2 \mathrm{C} 1$ & gi|375,314,775 & $66 \mathrm{kDa}$ & 2.01 \\
\hline 4 & Keratin, type I cytoskeletal 9 & $\mathrm{~K} 1 \mathrm{C9}$ & gi| $675,724,924$ & $62 \mathrm{kDa}$ & 1.84 \\
\hline 5 & Ubiquitin carboxyl-terminal hydrolase 3 & UBP3 & gi| $55,770,886$ & $59 \mathrm{kDa}$ & 1.54 \\
\hline 6 & Histamine N-methyltransferase & HNMT & gi|5,901,970 & $33 \mathrm{kDa}$ & 1.53 \\
\hline 7 & Vomeronasal type-1 receptor 5 & VN1R5 & gi|27,777,675 & $41 \mathrm{kDa}$ & 1.5 \\
\hline 8 & Kinesin-associated protein 3 & KIFAP3 & gi|18,105,054 & $91 \mathrm{kDa}$ & 1.47 \\
\hline 9 & Zinc finger protein 592 & ZN592 & gi|108,860,697 & 137 kDa & 1.47 \\
\hline 10 & Otogelin-like protein & OTOGL & gi|449,081,296 & 262 kDa & 1.46 \\
\hline 11 & Period circadian protein homolog 2 & PER2 & gi $\mid 6,683,645$ & 137 kDa & 1.43 \\
\hline 12 & Collagen alpha-3(IX) chain & CO9A3 & gi|426,392,464 & $63 \mathrm{kDa}$ & 1.39 \\
\hline 13 & RAS (RAD And GEM)-Like GTP-Binding 1 & REM1 & gi|724,948,224 & $33 \mathrm{kDa}$ & 1.39 \\
\hline 14 & Coiled-coil and C2 domain-containing protein 2A & C2D2A & gi|197,209,974 & 186 kDa & 1.37 \\
\hline 15 & Kinesin-like protein KIF12 & KIF12 & gi|85,541,032 & $71 \mathrm{kDa}$ & 1.36 \\
\hline 16 & Prostate stem cell antigen & PSCA & gi|114,622,040 & $13 \mathrm{kDa}$ & 1.36 \\
\hline 17 & Conserved oligomeric Golgi complex subunit 2 & COG2 & gi $\mid 6,678,676$ & $83 \mathrm{kDa}$ & 1.36 \\
\hline 18 & Adenomatous polyposis coli protein & APC & gi|53,759,122 & 312 kDa & 1.35 \\
\hline 19 & Elongation of very long chain fatty acids protein 7 & ELOV7 & gi|157,388,947 & 145 kDa & 1.34 \\
\hline 20 & SH3 domain-binding protein 4 & SH3B4 & gi|7,657,562 & 107 kDa & 1.34 \\
\hline 21 & Keratin, type II cytoskeletal 5 & $\mathrm{~K} 2 \mathrm{C} 5$ & gi|119,395,754 & $62 \mathrm{kDa}$ & 1.31 \\
\hline 22 & Retinoblastoma-associated protein & $\mathrm{RB}$ & gi|108,773,787 & 106 kDa & 1.31 \\
\hline 23 & Serine/threonine-protein kinase ULK3 & ULK3 & gi|656,214,624 & $31 \mathrm{kDa}$ & 1.3 \\
\hline 24 & Zinc finger protein 469 & ZN469 & gi|188,536,004 & $410 \mathrm{kDa}$ & 1.3 \\
\hline 25 & Kinetochore protein Spc25 & SPC25 & gi|10,190,716 & $26 \mathrm{kDa}$ & 1.3 \\
\hline 26 & Shugoshin 2 & $\mathrm{SGO} 2$ & gi|229,892,306 & 145 kDa & 1.3 \\
\hline 27 & POTE ankyrin domain family member I & POTEI & gi|475,808,427 & $121 \mathrm{kDa}$ & 1.29 \\
\hline 28 & Myelin expression factor 2 & MYEF2 & gi|296,439,294 & $64 \mathrm{kDa}$ & 1.28 \\
\hline 29 & Mucolipin-3 & MCLN3 & gi|24,496,763 & $64 \mathrm{kDa}$ & 1.28 \\
\hline 30 & Putative heat shock protein HSP 90-beta 4 & H90B4 & gi|61,104,915 & $56 \mathrm{kDa}$ & 1.28 \\
\hline 31 & U6 snRNA-associated Sm-like protein LSm4 & LSM4 & gi|513,024,372 & $15 \mathrm{kDa}$ & 1.28 \\
\hline 32 & tRNA (adenine(58)-N(1))-methyltransferase, mitochondrial & TR61B & gi|222,831,587 & $53 \mathrm{kDa}$ & 1.27 \\
\hline 33 & Phosphatidate phosphatase LPIN1 & LPIN1 & gi|387,528,011 & $99 \mathrm{kDa}$ & 1.27 \\
\hline 34 & Uncharacterized protein C1orf167 & CA167 & gi|158,706,475 & 162 kDa & 1.26 \\
\hline 35 & Nebulin & NEBU & gi|635,377,472 & 772 kDa & 1.26 \\
\hline 36 & SPRY domain-containing protein 3 & SPRY3 & gi|14,249,554 & $31 \mathrm{kDa}$ & 1.26 \\
\hline 37 & Nuclear receptor 2C2-associated protein & NR2CA & gi|28,882,043 & $16 \mathrm{kDa}$ & 1.26 \\
\hline 38 & Charged multivesicular body protein 5 & CHMP5 & gi|189,409,150 & $25 \mathrm{kDa}$ & 1.25 \\
\hline 39 & Collagen alpha- $5(\mathrm{VI})$ chain & CO6A5 & gi|189,082,691 & 290 kDa & 1.25 \\
\hline 40 & Dedicator of cytokinesis protein 11 & DOC11 & gi|145,699,123 & 215 kDa & 1.25 \\
\hline 41 & BPI fold-containing family A member 3 & BPIA3 & gi|109,627,654 & $28 \mathrm{kDa}$ & 1.25 \\
\hline 42 & Obscurin & OBSCN & gi|403,501,446 & $925 \mathrm{kDa}$ & 1.24 \\
\hline 43 & CCR4-NOT transcription complex subunit 6 & CNOT6 & gi|28,872,863 & $63 \mathrm{kDa}$ & 1.24 \\
\hline 44 & 5-methylcytosine rRNA methyltransferase NSUN4 & NSUN4 & gi| $40,316,918$ & $49 \mathrm{kDa}$ & 1.23 \\
\hline
\end{tabular}


Table 1 iTRAQ ratios of up-regulated proteins in GC tissues (Continued)

\begin{tabular}{|c|c|c|c|c|c|}
\hline No. & Protein name & Gene name & Accession Number & Molecular Weight & Ratio \\
\hline 45 & Gamma-interferon-inducible lysosomal thiol reductase & GILT & gi|6,165,618 & $28 \mathrm{kDa}$ & 1.23 \\
\hline 46 & POZ-, AT hook-, and zinc finger-containing protein 1 & PATZ1 & gi|586,477,589 & $70 \mathrm{kDa}$ & 1.23 \\
\hline 47 & Suppressor of zest 12 & SUZ12 & gi|197,333,809 & $83 \mathrm{kDa}$ & 1.23 \\
\hline 48 & MICOS Complex Subunit MIC27 & MIC27 & gi|116,812,610 & $29 \mathrm{kDa}$ & 1.22 \\
\hline 49 & SPARC-related modular calcium-binding protein 1 & SMOC1 & gi|11,545,873 & $48 \mathrm{kDa}$ & 1.22 \\
\hline 50 & Replication Termination Factor 2 & RTF2 & gi|193,788,722 & $34 \mathrm{kDa}$ & 1.22 \\
\hline 51 & Poly(A) polymerase alpha & PAPOA & gi|32,490,557 & $83 \mathrm{kDa}$ & 1.22 \\
\hline 52 & RNA-binding protein 42 & RBM42 & gi|545,830,327 & $50 \mathrm{kDa}$ & 1.22 \\
\hline 53 & Platelet-activating factor acetylhydrolase IB subunit beta & PAFAH1B2 & gi| $532,006,944$ & $26 \mathrm{kDa}$ & 1.21 \\
\hline 54 & Ornithine decarboxylase antizyme 1 & OAZ1 & gi|576,067,926 & $25 \mathrm{kDa}$ & 1.21 \\
\hline 55 & Zinc finger and BTB domain-containing protein 11 & ZBT11 & gi|166,235,167 & $119 \mathrm{kDa}$ & 1.21 \\
\hline 56 & Methyltransferase-like protein 17, mitochondrial & MET17 & gi|12,232,389 & $50 \mathrm{kDa}$ & 1.21 \\
\hline 57 & Glycogen synthase kinase-3 beta & GSK3B & gi|395,758,596 & $48 \mathrm{kDa}$ & 1.21 \\
\hline 58 & Thymosin beta-4 & TYB4 & gi|112,180,578 & $5 \mathrm{kDa}$ & 1.21 \\
\hline 59 & Chromosome transmission fidelity protein 18 homolog & CTF18 & gi|27,501,458 & $129 \mathrm{kDa}$ & 1.21 \\
\hline
\end{tabular}

To address whether and how USP3 stabilizes SUZ12, we treated vector and BCG-823 cells expressing USP3 with cycloheximide and prepared extracts after different time points (Fig. 5c). The SUZ12 protein level was decreased in vector cells with a half-life of approximately $3.8 \mathrm{~h}$, which demonstrated that the protein was subjected to proteolysis. In contrast, the protein was essentially stable over the entire time period in cells expressing USP3 (estimated half-life of SUZ12: $8.4 \mathrm{~h}$ ). These results suggested that USP3 enhanced SUZ12 stability by blocking protein degradation.

Since USP3 mediates the removal and processing of ubiquitin [26], it was speculated that USP3 directly regulates SUZ12 protein stability through deubiquitination of SUZ12. To assess this possibility, flag-tagged USP3 or empty vector plasmid was transfected into BCG-823 cells. Western blot showed that the ubiquitination of SUZ12 was strongly inhibited by USP3 expression in the presence or absence of MG132, a potent inhibitor of the $26 \mathrm{~S}$ proteasome (Fig. 5d). Knockdown of USP3 expression led to opposite results (Fig. 5e).

Taken together, these findings suggest that USP3 is a DUB that controls the level of SUZ12 protein through interaction with and deubiquitination of SUZ12.

\section{SUZ12 is involved in USP3-induced GC cell migration, invasion and EMT}

To investigate the relationship among USP3, SUZ12 and the capacity for cell migration and invasion, the expression of SUZ12 in GC cells was downregulated by siRNA 1 (Fig. 6a). Intriguingly, the cell migratory and invasive abilities of GC cells transfected with SUZ12 siRNA 1 were significantly weakened (Fig. 6b \& c).
Recently, it has been shown that upregulation of SUZ12 promotes metastasis through the regulation of EMT in cancer cells $[16,17]$. We thus investigated whether SUZ12 affects the EMT process by regulating USP3 expression. We have indicated that USP3-transfected cells exhibited an elongated fibroblastoid phenotype by phase-contrast microscopy. However, SUZ12 knockdown in USP3 cells restored their normal phenotype, which consists of a round or flat morphology with short cytoplasmic processes (Fig. 6d). Furthermore, USP3 and SUZ12 expression was detected by IHC in metastatic tissues obtained from lymph nodes. We found that USP3 and SUZ12 were expressed at high levels in the nucleus and cytoplasm of cancer cells from two patients (Fig. 6e).

In addition, we performed a Western blot analysis and showed that knockdown of SUZ12 in USP3-overexpressing cells increased the expression of an epithelial marker (Ecadherin) but decreased the expression of mesenchymal markers (vimentin, Snail and MMP9) (Fig. 6f).

Taken together, these data suggest that the USP3-SUZ12 axis promotes an EMT-like phenotype in GC cells.

\section{Positive correlation between USP3 and SUZ12 expression in GC cells and GC tissues}

To further examine the relationship between USP3 and SUZ12, the expression of USP3 and SUZ12 was analyzed in GC cells and tissues. The results showed that the protein levels of USP3 and SUZ12 were positively correlated in the majority of these GC cells (Fig. 7a). Immunohistochemical staining showed that USP3- and SUZ12-positive signals were strongly or moderately expressed in the carcinoma cells of all ten 


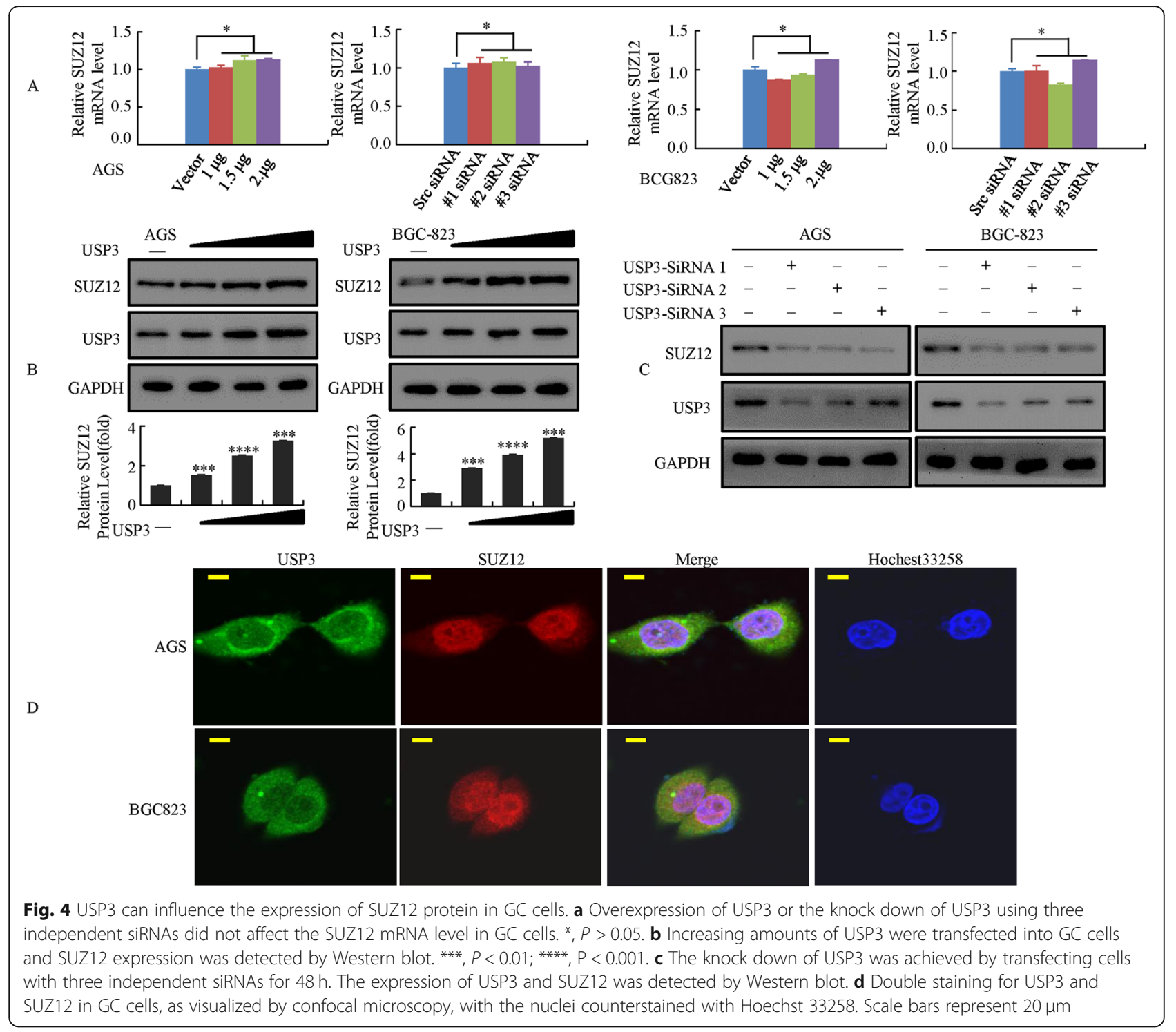

GC samples as exemplified in Fig. 7b. Moreover, the expression profiles of USP3 and SUZ12 in cancerous and normal gastric tissues were inversely correlated with the profile of the epithelial marker E-cadherin (Fig. 7b). USP3 and SUZ12 were only expressed in cancer cells, whereas E-cadherin was predominantly expressed in normal epithelial cells (Fig. 7b). In addition, Spearman's rank correlation analysis confirmed that USP3 protein expression was positively associated with SUZ12 protein expression in human tissue (Fig. 7c). The levels of USP3 and SUZ12 proteins were negatively correlated with the levels of Ecadherin protein (Fig. $7 d$ ).

Together, these results demonstrate a positive correlation between USP3 and SUZ12 expression levels and a negative correlation between the levels of USP3/SUZ12 and E-cadherin protein.

\section{Discussion}

In this study, we report on USP3, a gene that is overexpressed in various cancers, including GC and that acts as an important oncogene. USP3 increased cell migration, invasion and the ectopic expression of USP3 in GC cells and induced EMT in vitro and in vivo. Moreover, USP3 interacts with, depolyubiquitylates and stabilizes SUZ12. Co-expression of USP3 and SUZ12 induces cell metastasis through the induction of EMT. These findings demonstrate that USP3 is an essential regulator of SUZ12 and that the USP3SUZ12 signaling axis plays a critical role in the progression and metastasis of GC.

USP3 is a member of the ubiquitin-specific protease (USP) subfamily of deubiquitinating enzymes (DUBs) [8-10]. Previous studies have revealed its potential roles in the molecular pathophysiology of cancer. Here, we 


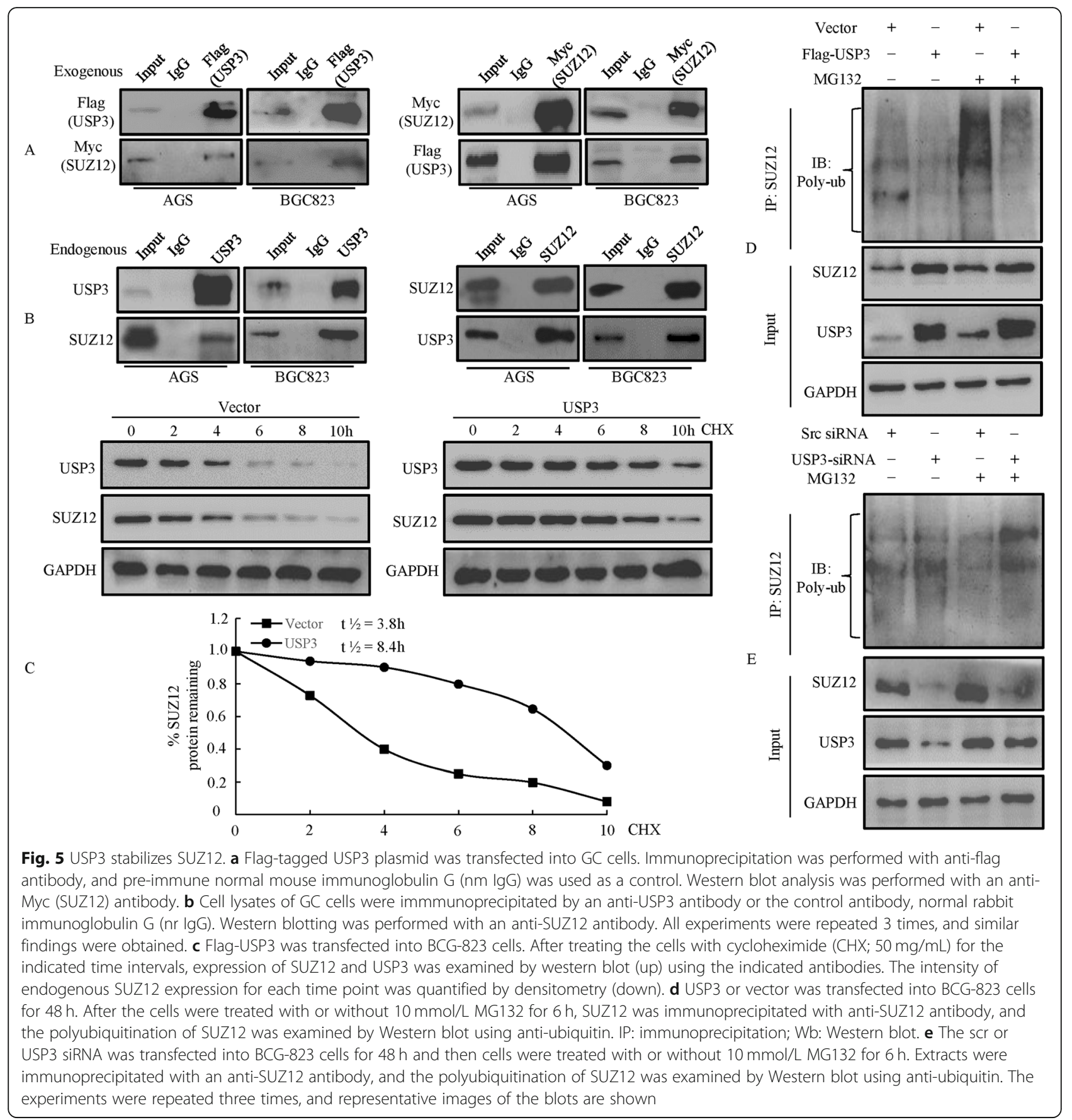

provided evidence that the GC tissues had higher USP3 expression relative to adjacent adjacent nontumor tissues, which further confirmed the findings of previous studies. A significant relationship was observed between elevated USP3 expression and differentiation, AJCC stage and lymph node metastasis. Moreover, high expression of USP3 predicted poor survival in GC patients according to a survival analysis. In addition, USP3 overexpression promoted GC cell migration and invasiveness. These findings indicated a potential relationship between USP3 expression and an aggressive phenotype in GC.

EMT is implicated in the initial step of cancer metastasis and is indispensable for cancer cells to break through the barriers formed by normal tissues so that a tumor can metastasize $[20,23,27]$. The heterogeneity in GC also increases the risk of gastric carcinoma progression and metastasis and exacerbates therapeutic resistance [28]. Recently, of the proteins in the ubiquitin-specific protease (USP) family, USP10 was 


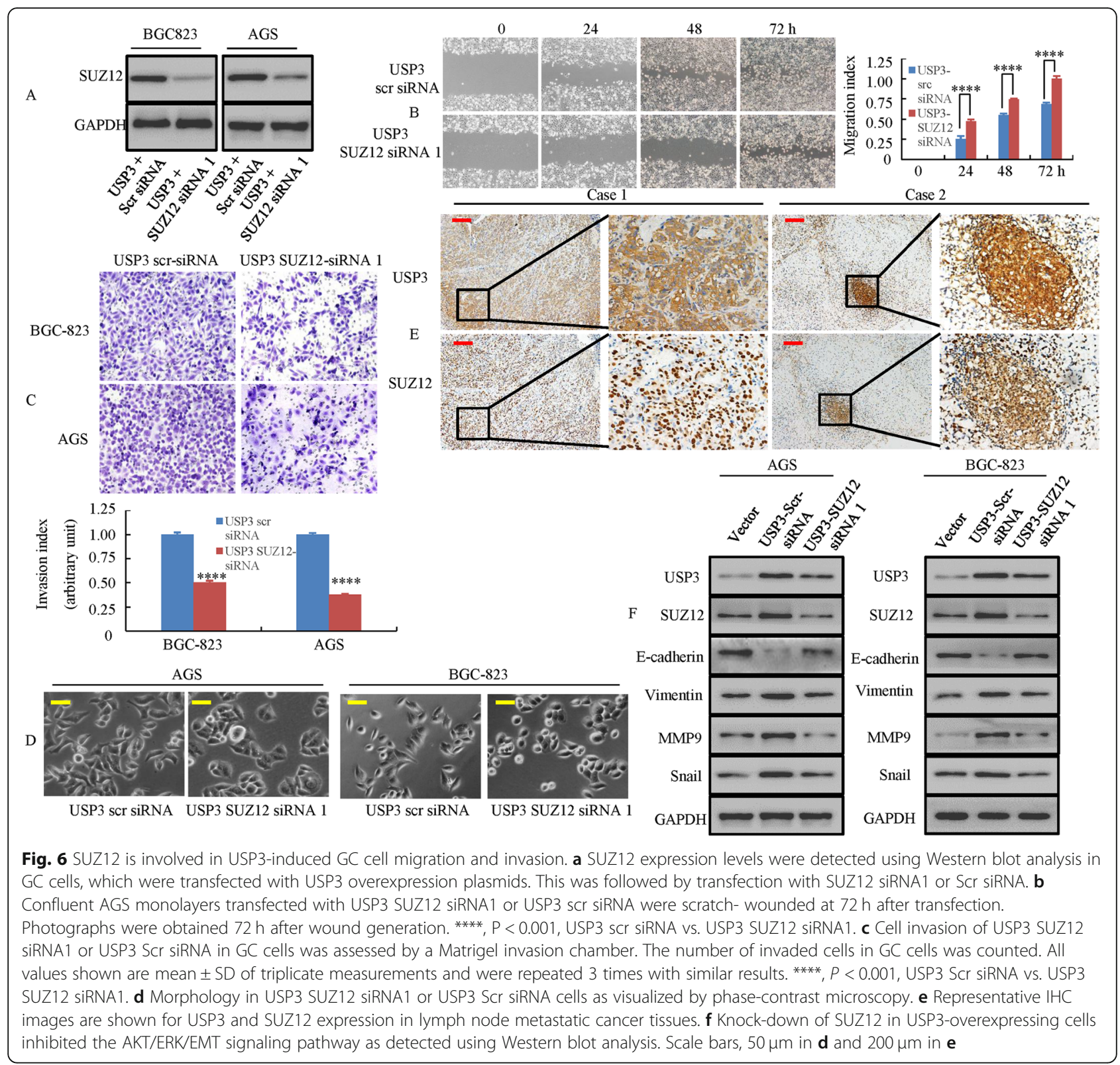

found to induce EMT and aggressiveness in human cancer [29, 30]. Consistently, we have shown that USP3 overexpression caused the loss of epithelial polarity and the expression of EMT markers including the following: loss of the epithelial cell marker Ecadherin and upregulation of the mesenchymal cell marker vimentin. Moreover, ectopic expression of USP3 led to the acquisition of molecular and functional characteristics of EMT, which caused the cells to appear scattered. This phenomenon is consistent with the previous theory that EMT is essential for the dissemination of tumor cells from adjacent tissues and for the seeding of new tumors at distant sites [31]. Hence, our study provides significant insights into the novel roles of USP3 as a factor that promotes GC metastasis through EMT.

TGF- $\beta 1$ is a multifunctional cytokine that regulates a wide range of cellular functions. TGF- $\beta 1$-stimulates cells to become spindle-shaped and undergo morphological changes such as a decrease in cell-cell adhesion and loss of polarity [24, 25]. TGF- $\beta 1$ is thought to be a key factor that contributes to cancer progression, primarily via EMT-triggered metastasis [25, 32]. Studies have reported that some members of the USP gene family have a role in mediating TGF- $\beta$-induced EMT. Kit Leng Lui $S$ et al. showed that USP2 6 regulates TGF- $\beta$ signaling by deubiquitinating and stabilizing SMAD7 [33]. Moreover, TGF$\beta$ upregulates the translation of USP15 via the PI3K/ 


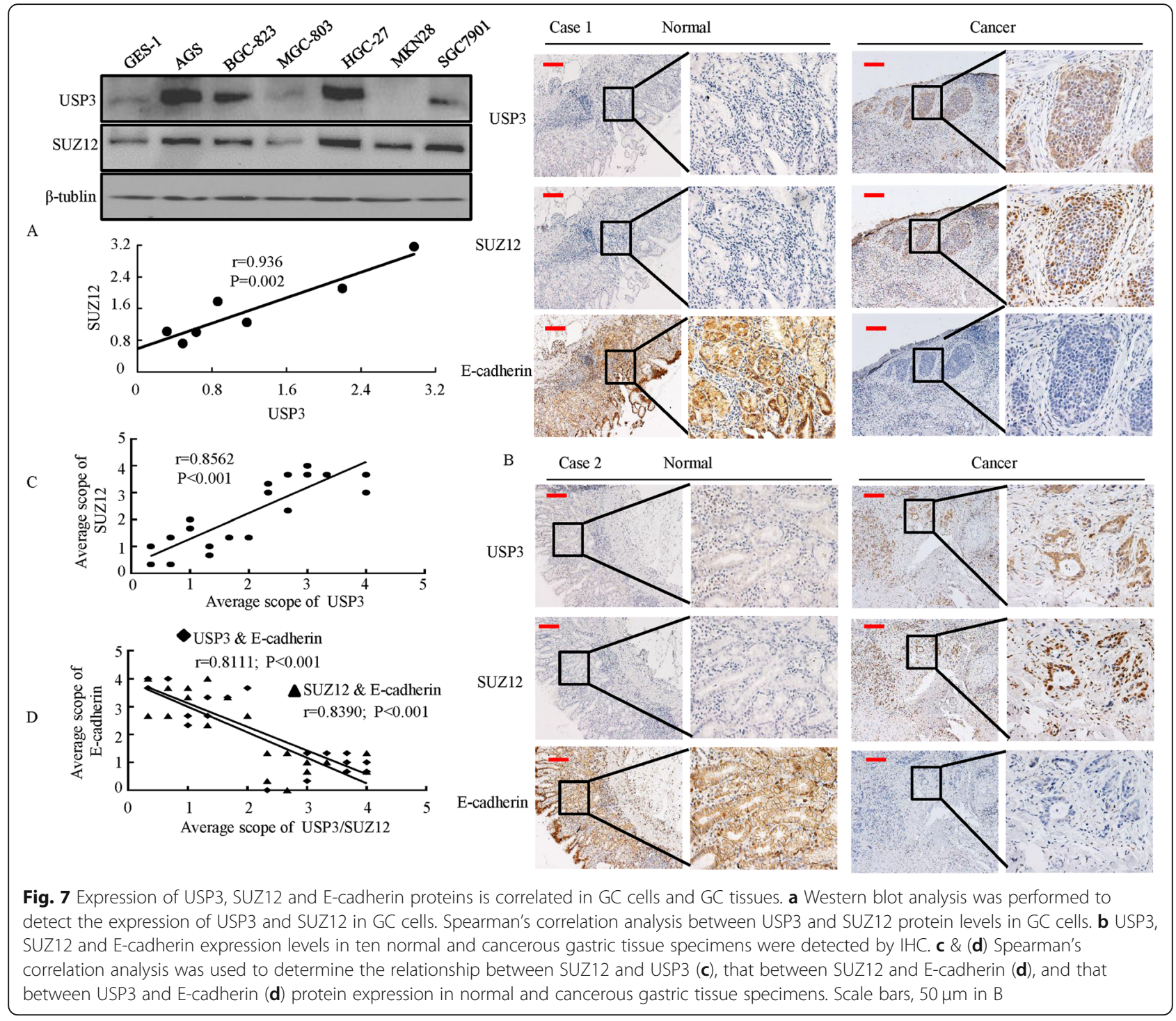

AKT pathway to promote p53 stability [34]. Among the USP genes, we found that induction of EMT in GC cells by TGF- $\beta 1$ was associated with a significant increase in USP3 expression. When USP3 expression was silenced, the EMT phenotype induced by TGF- $\beta 1$ was abrogated, and USP3 expression was also decreased. This is the first study that has explored the effect of USP3 on TGF- $\beta 1$ induced EMT in GC.

To better understand the effect of USP3 on quantitative protein changes in GC, we utilized differential proteomics, which consisted of Isobaric Tag for Relative and Absolute Quantitation (iTRAQ) in combination with LC-MS/MS. iTRAQ has gained popularity for its ability to perform concurrent identification and relative quantification of hundreds of proteins; iTRAQ has also been employed in several oncoproteomics studies [35-37]. In the current study, iTRAQ data showed that the differentially expressed proteins $(59$ upregulated and 63 downregulated) were widely distributed in the cell and demonstrated that they played a role in all aspects of cell biology, which indicates that USP3 is involved in almost all biological processes.

As mentioned above, SUZ12 was chosen for further study. SUZ12, a core component of the Polycomb PRC2-HMTase complex, plays an oncogenic role in GC [11, 12]. Previous studies have indicated that overexpression of SUZ12 is associated with migration and invasion through its regulation of EMT in tumors $[16,17]$. Recently, researchers have shown that SUZ12 was transcriptionally regulated by some proteins, including NF- $\mathrm{kB}$ and E2F1 [38]. However, the mechanism by which SUZ12 protein stability and turnover are regulated remains unknown. In the current study, SUZ12 was identified as one of USP3interacting proteins, which was confirmed by co-IP and confocal laser scanning microscopy analysis. 
Sharma $\mathrm{N}$ et al. have found that USP3 counteracts RNF168 via deubiquitinating $\mathrm{H} 2 \mathrm{~A}$ and $\gamma \mathrm{H} 2 \mathrm{AX}$ at lysines 13 and 15 [39]. Fu S and his colleagues have shown that USP3 interacts with p53 and regulates p53 stability and that depletion of USP3 leads to accelerated degradation of p53 in normal cells and therefore enhanced cell proliferation and transformation [40]. Consistently, overexpression of USP3 stabilizes the SUZ12 protein by binding to and deubiquitinating SUZ12 and subsequently promoting GC progression. These findings are consistent with the hypothesis that regulation of SUZ12 by USP3 is a posttranslational event.

To determine the relationship between expression of USP3 and SUZ12, SUZ12 was downregulated in USP3overexpressing cells using siRNA. Intriguingly, the knock down of SUZ12 reduced the capability of cell migration and invasion compared with the USP3-expressing cells, which suggests that USP3 exerted its effects at least in part by deubiquitinating and stabilizing SUZ12. Moreover, SUZ12 knockdown in USP3-expressing cells restored their normal morphology and inhibited the expression of vimentin, Snail and MMP-2/9. Thus, the results suggest that the induction of EMT by SUZ12 is mediated by USP3. In addition, we observed that the distribution pattern of USP3 was highly congruent with that of SUZ12 in GC cells and clinical GC samples. For this reason, it is hypothesized that USP3 drives GC invasion, metastasis and EMT through a mechanism wherein USP3 stabilizes SUZ12 protein.

\section{Conclusion}

In conclusion, our study shows that USP3 could promote cell migration, invasion and EMT in GC cells through interacting with and deubiquitinating SUZ12. Thus, targeting the USP3-SUZ12 axis may provide a potential therapeutic target for the treatment of GC.

\section{Additional files}

Additional file 1: Table S1. Correlation between USP3 protein expression and the clinicopathological parameters of GC. Table S2. ITRAQ ratios of down-regulated proteins in GC tissues. Supplementary materials and methods. (DOCX $53 \mathrm{~kb}$ )

Additional file 2: Figure S1. Functional analysis of USP3 in vitro. (A) Invasive potential of the GC cells transfected with the USP3 or Vector. **, $P<0.05$; ****, $P<0.001$. (B) (C) \& (E) For the wound-healing experiments, cells were analyzed with live-cell microscopy. ${ }^{* * * *}, P<0.001$. (D) Invasive potential of the GC cells transfected with USP3 siRNA or $\operatorname{src}$ siRNA. ***** $P<0.001$. (F) USP3 is regulated by the AKT/ERK/EMT signaling pathway in GC cells according to the Western blot analysis. (TIF $808 \mathrm{~kb}$ )

Additional file 3: Figure S2. Overexpression of USP3 enhances TGF- $\beta 1$ induced EMT. (A and B) BCG-823 cells were pretreated with various concentrations of TGF- $\beta 1(0-2 \mathrm{ng} / \mathrm{ml})$ for $48 \mathrm{~h}$ (A) or with TGF- $\beta 1(1 \mathrm{ng} / \mathrm{ml})$ for $48 \mathrm{~h}$ (B) and then subjected to western blot to detect $\mathrm{E}$-cadherin, vimentin and USP3. GAPDH was used as the internal control. (C) BCG-823 cells were treated with recombinant TGF- $\beta 1(2 \mathrm{ng} / \mathrm{ml})$ in the presence of neutralizing anti-TGF- $\beta 1$ antibody (a-TGF- $\beta, 2 \mathrm{mg} / \mathrm{ml}$ ) or mouse lgG
(mlgG) for $48 \mathrm{~h}$. (D) Cells in which USP3 was knocked down or transfected with scr siRNA were treated with g-TGF- $\beta 1$ for an additional $48 \mathrm{~h}$. The expression of USP3, E-cadherin and vimentin was detected by Western blot. (E) The morphology of GC cells was observed under an inverted microscope. (F) The GC cells were transfected with USP3 siRNA or scr siRNA for $24 \mathrm{~h}$ followed by TGF- $\beta 1$ treatment for $24 \mathrm{~h}$. Representative images and data from a Transwell assay in GC cells. Each bar represents the mean \pm SD. ${ }^{* * *}, P<0.001$, compared with GC cells treated with TGF- $\beta 1$. ****, $P<0.001$, compared with cells transfected with USP3 siRNA and treated with TGF- $\beta 1$. The error bars represent the mean \pm SD from 3 independent experiments. Scale bars, $50 \mu \mathrm{m}$ in E. (TIF $4576 \mathrm{~kb}$ )

\section{Abbreviations}

ATCC: American Type Culture Collection; DUBs: deubiquitinating enzymes; EMT: epithelial-to-mesenchymal transition; IHC: immunohistochemistry; iTRAQ: Isobaric Tag for Relative and Absolute Quantitation; siRNA: short interfering RNA; SUZ12: suppressor of zeste 12 protein homolog; USP3: ubiquitin-specific protease 3

\section{Acknowledgments}

We acknowledge the generous support of the Guangdong Provincial Key Laboratory of Gastroenterology, Department of Gastroenterology, Nanfang Hospital, Southern Medical University.

\section{Authors' contributions}

This study was designed and conceived by JW, SL and LX. Experiments in vitro was performed by XW, ML, HZ, JW, YX, WD and JL. Experiments in vivo were conducted by XW, JW, HZ, WT, YX. DZ, ML and WZ helped with data analysis. YS, YZ and YC were responsible for the collection of specimens. GL and $\mathrm{AL}$ contributed to technical support. LX, SL supervised the project and JW wrote the manuscript. All authors read and approved the final manuscript.

\section{Funding}

This study was supported by grants from the National Natural Science Funds of China (No.81672875 and 81772964); Special Foundation for Economic and Technological Development of Longgang District, Shenzhen (NO: LGKCYLWS2018000160 and NO: LGKCYLWS2018000141). The funding organization: the Guangzhou Pilot Project of the Clinical and Translational Research Center (early gastrointestinal cancer, No.7415696196402).

\section{Availability of data and materials}

All remaining data are available within the article and supplementary files, or available from the authors upon request.

\section{Ethics approval and consent to participate}

This study was reviewed and approved by the Medical Ethics Committee of Nanfang Hosital (NFEC-2017-062), Southern Medical University, Guangzhou, China. All animal studies were approved by the Institutional Animal Care and Use Committee of Committee of Nanfang Hosital.

Consent for publication

All authors have agreed to publish this manuscript.

Competing interests

The authors declare that they have no competing interests.

\section{Author details}

${ }^{1}$ Guangdong Provincial Key Laboratory of Gastroenterology, Department of Gastroenterology, Nanfang Hospital, Southern Medical University, Guangzhou 510515, China. ${ }^{2}$ Department of Clinical Laboratory, General Hospital of Southern Theatre Command, Guangzhou 510010, China. ${ }^{3}$ Department of Gastroenterology, Longgang District People's Hospital, Shenzhen 518172, China. ${ }^{4}$ Department of Medical Oncology, the First people's Hospital of Yunnan Province, Medical School of Kunming University of Science and Technology, Kunming 650032, China. ${ }^{5}$ Department of Gastroenterology, The third affiliated Hospital of Guangzhou Medical University, Guangzhou 510150, China. ${ }^{6}$ Department of General Surgery, Nanfang Hospital, Southern Medical University, Guangzhou 510515, China. ${ }^{7}$ Department of Digestive Medicine, 
Nanfang Hospital, Southern Medical University, Guangzhou 510515 Guangdong Province, China.

\section{Received: 4 February 2019 Accepted: 5 June 2019}

\section{Published online: 24 June 2019}

\section{References}

1. Balakrishnan M, George R, Sharma A, Graham DY. Changing trends in stomach Cancer throughout the world. Current gastroenterology reports. 2017;19(8):36.

2. Huang $T$, Zhou $Y$, Cheng AS, Yu J, To KF, Kang W. NOTCH receptors in gastric and other gastrointestinal cancers: oncogenes or tumor suppressors? Mol Cancer. 2016;15(1):80.

3. Uen YH, Fang CL, Lin CC, Hseu YC, Hung ST, Sun DP, et al. Ceramide synthase 6 predicts the prognosis of human gastric cancer: it functions as an oncoprotein by dysregulating the SOCS2/JAK2/STAT3 pathway. Mol Carcinog. 2018;57(12):1675-89.

4. Yan C, Su H, Song X, Cao H, Kong L, Cui W. Smad ubiquitination regulatory factor 1 (Smurf1) promotes thyroid Cancer cell proliferation and migration via ubiquitin-dependent degradation of Kisspeptin-1. Cellular physiology and biochemistry: international journal of experimental cellular physiology, biochemistry, and pharmacology. 2018:49(5):2047-59.

5. Bedekovics T, Hussain S, Feldman AL, Galardy PJ. UCH-L1 is induced in germinal center B cells and identifies patients with aggressive germinal center diffuse large B-cell lymphoma. Blood. 2016;127(12):1564-74.

6. Tsou WL, Sheedlo MJ, Morrow ME, Blount JR, McGregor KM, Das C, et al. Systematic analysis of the physiological importance of deubiquitinating enzymes. PloS One. 2012;7(8):e43112.

7. Peschiaroli A, Skaar JR, Pagano M, Melino G. The ubiquitin-specific protease USP47 is a novel beta-TRCP interactor regulating cell survival. Oncogene. 2010:29(9):1384-93.

8. Fang CL, Lin CC, Chen HK, Hseu YC, Hung ST, Sun DP, et al. Ubiquitinspecific protease 3 overexpression promotes gastric carcinogenesis and is predictive of poor patient prognosis. Cancer Sci. 2018;109(11):3438-49.

9. Cheng YC, Shieh SY. Deubiquitinating enzyme USP3 controls CHK1 chromatin association and activation. Proc Natl Acad Sci U S A. 2018; 115(21):5546-51.

10. Cui J, Song Y, Li Y, Zhu Q, Tan P, Qin Y, et al. USP3 inhibits type I interferon signaling by deubiquitinating RIG-I-like receptors. Cell Res. 2014;24(4):400-16.

11. Vizan P, Beringer M, Ballare C, Di Croce L. Role of PRC2-associated factors in stem cells and disease. FEBS J. 2015;282(9):1723-35.

12. Lee SR, Roh YG, Kim SK, Lee JS, Seol SY, Lee HH, et al. Activation of EZH2 and SUZ12 regulated by E2F1 predicts the disease progression and aggressive characteristics of bladder Cancer. Clinical cancer research: an official journal of the American Association for Cancer Research. 2015;21(23): 5391-403.

13. Benoit YD, Witherspoon MS, Laursen KB, Guezguez A, Beausejour M, Beaulieu JF, et al. Pharmacological inhibition of polycomb repressive complex-2 activity induces apoptosis in human colon cancer stem cells. Exp Cell Res. 2013:319(10):1463-70.

14. Kim HG, Kim DJ, Li S, Lee KY, Li X, Bode AM, et al. Polycomb (PcG) proteins, BMI1 and SUZ12, regulate arsenic-induced cell transformation. J Biol Chem. 2012:287(38):31920-8

15. Cui Y, Chen J, He Z, Xiao Y. SUZ12 depletion suppresses the proliferation of gastric cancer cells. Cellular physiology and biochemistry: international journal of experimental cellular physiology, biochemistry, and pharmacology. 2013;31(6):778-84.

16. Hwang-Verslues WW, Chang PH, Jeng YM, Kuo WH, Chiang PH, Chang YC, et al. Loss of corepressor PER2 under hypoxia up-regulates OCT1-mediated EMT gene expression and enhances tumor malignancy. Proc Natl Acad Sci U S A. 2013;110(30):12331-6.

17. Xia R, Jin FY, Lu K, Wan L, Xie M, Xu TP, et al. SUZ12 promotes gastric cancer cell proliferation and metastasis by regulating KLF2 and E-cadherin. Tumour biology: the journal of the International Society for Oncodevelopmental Biology and Medicine. 2015;36(7):5341-51.

18. Wu Y, Peng $Y$, Wu M, Zhang $W$, Zhang $M$, Xie $R$, et al. Oncogene FOXK1 enhances invasion of colorectal carcinoma by inducing epithelialmesenchymal transition. Oncotarget. 2016:7(32):51150-62.

19. Xie R, Wang J, Tang W, Li Y, Peng Y, Zhang H, et al. Rufy3 promotes metastasis through epithelial-mesenchymal transition in colorectal cancer. Cancer Lett. 2017:390:30-8.
20. Zhang $H$, Wu X, Xiao $Y$, Wu L, Peng $Y$, Tang W, et al. Coexpression of FOXK1 and vimentin promotes EMT, migration, and invasion in gastric cancer cells. J Mol Med. 2019;97(2):163-76.

21. Hu W, Xu W, Shi Y, Dai W. IncRNA HOTAIR upregulates COX-2 expression to promote invasion and migration of nasopharyngeal carcinoma by interacting with miR-101. Biochem Biophys Res Commun. 2018;505(4):1090-6.

22. Li Y, Zhu X, Zeng Y, Wang J, Zhang X, Ding YQ, et al. FMNL2 enhances invasion of colorectal carcinoma by inducing epithelial-mesenchymal transition. Mol Cancer Res. 2010;8(12):1579-90.

23. Li Q, Tang H, Hu F, Qin C. Knockdown of A-kinase anchor protein 4 inhibits hypoxia-induced epithelial-to-mesenchymal transition via suppression of the Wnt/beta-catenin pathway in human gastric cancer cells. J Cell Biochem. 2018;119(12):10013-20

24. Liu Z, Li Q, Li K, Chen L, Li W, Hou M, et al. Telomerase reverse transcriptase promotes epithelial-mesenchymal transition and stem cell-like traits in cancer cells. Oncogene. 2013;32(36):4203-13.

25. Peng $Y$, Zhang $P$, Huang $X$, Yan Q, Wu M, Xie R, et al. Direct regulation of FOXK1 by C-Jun promotes proliferation, invasion and metastasis in gastric cancer cells. Cell Death Dis. 2016;7(11):e2480.

26. Lancini C, van den Berk PC, Vissers JH, Gargiulo G, Song JY, Hulsman D, et al. Tight regulation of ubiquitin-mediated DNA damage response by USP3 preserves the functional integrity of hematopoietic stem cells. J Exp Med. 2014;211(9):1759-77.

27. Zhang $P$, Tang WM, Zhang H, Li YQ, Peng Y, Wang J, et al. MiR-646 inhibited cell proliferation and EMT-induced metastasis by targeting FOXK1 in gastric cancer. Br J Cancer. 2017;117(4):525-34.

28. Santos JC, Carrasco-Garcia E, Garcia-Puga M, Aldaz P, Montes M, FernandezReyes $M$, et al. SOX9 elevation acts with canonical WNT signaling to drive gastric Cancer progression. Cancer Res. 2016;76(22):6735-46.

29. Ouchida AT, Kacal M, Zheng A, Ambroise G, Zhang B, Norberg E, et al. USP10 regulates the stability of the EMT-transcription factor slug/SNAI2. Biochem Biophys Res Commun. 2018;502(4):429-34.

30. Hou K, Zhu Z, Wang Y, Zhang C, Yu S, Zhu Q, et al. Overexpression and biological function of ubiquitin-specific protease 42 in gastric Cancer. PLoS One. 2016;11(3):e0152997.

31. Yilmaz M, Christofori G. EMT, the cytoskeleton, and cancer cell invasion. Cancer Metastasis Rev. 2009;28(1-2):15-33.

32. Wu X, Zhao J, Ruan Y, Sun L, Xu C, Jiang H. Sialyltransferase ST3GAL1 promotes cell migration, invasion, and TGF-beta1-induced EMT and confers paclitaxel resistance in ovarian cancer. Cell Death Dis. 2018;9(11):1102.

33. Kit Leng Lui $S$, Iyengar PV, Jaynes $P$, Isa Z, Pang B, Tan TZ, et al. USP26 regulates TGF-beta signaling by deubiquitinating and stabilizing SMAD7. EMBO Rep. 2017;18(5):797-808.

34. Liu WT, Huang KY, Lu MC, Huang HL, Chen CY, Cheng YL, et al. TGF-beta upregulates the translation of USP15 via the PI3KVAKT pathway to promote p53 stability. Oncogene. 2017;36(19):2715-23.

35. Sun S, Lee NP, Poon RT, Fan ST, He QY, Lau GK, et al. Oncoproteomics of hepatocellular carcinoma: from cancer markers' discovery to functional pathways. Liver international: official journal of the International Association for the Study of the Liver. 2007;27(8):1021-38.

36. Fang $Y$, Fu D, Tang W, Cai Y, Ma D, Wang H, et al. Ubiquitin C-terminal hydrolase 37, a novel predictor for hepatocellular carcinoma recurrence, promotes cell migration and invasion via interacting and deubiquitinating PRP19. Biochim Biophys Acta. 2013;1833(3):559-72.

37. Matta A, DeSouza LV, Shukla NK, Gupta SD, Ralhan R, Siu KW. Prognostic significance of head-and-neck cancer biomarkers previously discovered and identified using iTRAQ-labeling and multidimensional liquid chromatographytandem mass spectrometry. J Proteome Res. 2008;7(5):2078-87.

38. Su SK, Li CY, Lei PJ, Wang X, Zhao OY, Cai Y, et al. The EZH1-SUZ12 complex positively regulates the transcription of NF-kappaB target genes through interaction with UXT. J Cell Sci. 2016;129(12):2343-53.

39. Sharma N, Zhu Q, Wani G, He J, Wang QE, Wani AA. USP3 counteracts RNF168 via deubiquitinating $\mathrm{H} 2 \mathrm{~A}$ and gammaH2AX at lysine 13 and 15. Cell Cycle. 2014;13(1):106-14.

40. Fu S, Shao S, Wang L, Liu H, Hou H, Wang Y, et al. USP3 stabilizes p53 protein through its deubiquitinase activity. Biochem Biophys Res Commun. 2017:492(2):178-83.

\section{Publisher's Note}

Springer Nature remains neutral with regard to jurisdictional claims in published maps and institutional affiliations. 